\title{
The Cellular and Subcellular Localization of Huntingtin-Associated Protein 1 (HAP1): Comparison with Huntingtin in Rat and Human
}

\author{
Claire-Anne Gutekunst, ${ }^{1}$ Shi-Hua Li, ${ }^{2}$ Hong Yi, ${ }^{1}$ Robert J. Ferrante, ${ }^{3}$ Xiao-Jiang Li, ${ }^{2}$ and Steven M. Hersch ${ }^{1}$ \\ Departments of ${ }^{1}$ Neurology and ${ }^{2}$ Genetics, Emory University School of Medicine, Atlanta, Georgia 30329, and ${ }^{3}$ Geriatric \\ Research Education Clinical Center, Bedford Veterans Administration Medical Center, Bedford, Massachusetts 01730, \\ and Department of Neurology, Boston University School of Medicine, Boston, Massachusetts 02118
}

The cellular and subcellular distribution of HAP1 was examined in rat brain by light and electron microscopic immunocytochemistry and subcellular fractionation. HAP1 localization was also determined in human postmortem tissue from control and Huntington's disease (HD) cases by light microscopic immunocytochemistry. At the cellular level, the heterogeneity of HAP1 expression was similar to that of huntingtin; however, HAP1 immunoreactivity was more widespread. The subcellular distribution of HAP1 was examined using immunogold electron microscopy. Like huntingtin, HAP1 is a cytoplasmic protein that associates with microtubules and many types of membranous organelles, including mitochondria, endoplasmic reticulum, tubulovesicles, endosomal and lysosomal organelles, and synaptic vesicles. A quantitative comparison of the organelle associations of HAP1 and huntingtin showed them to be almost identical. Within HAP1-immunoreactive neurons in rat and human brain, populations of large and small immunoreactive puncta were visible by light microscopy. The large puncta, which were especially evident in the ventral forebrain, were intensely HAP1 immunoreactive. Electron microscopic analysis revealed them to be a type of nucleolus-like body, which has been named a stigmoid body, that may play a role in protein synthesis. The small puncta, less intensely labeled, were primarily mitochondria. These results indicate that the localization of HAP1 and huntingtin is more similar than previously appreciated and provide further evidence that HAP1 and huntingtin have localizations consistent with roles in intracellular transport. Our data also suggest, however, that HAP1 is not present in the abnormal intranuclear and neuritic aggregates containing the $\mathrm{N}$-terminal fragment of mutant huntingtin that are found in HD brains.

Key words: Huntington's disease; electron microscopy; immunogold; nucleolus-like bodies; cytoplasmic inclusions; stigmoid bodies
The neuropathology of Huntington's disease (HD) includes the selective loss of neurons that is most severe in the neostriatum but also affects other brain regions. Excitotoxic, oxidative, and apoptotic mechanisms of neuronal death have all been implicated in HD (Hersch and Ferrante, 1997; Wellington et al., 1997); however, none have yet been directly connected to the polyglutamine expansion occurring in the expressed protein huntingtin. In human neostriatum, neurons most vulnerable to neurodegeneration express the highest levels of huntingtin, whereas the resistant ones express little or no huntingtin (Gutekunst et al., 1995; Ferrante et al., 1997). Although the normal function of huntingtin is unknown, a role in intracellular transport has been hypothesized based on its subcellular association with microtubules and membrane-bound organelles (DiFiglia et al., 1995; Gutekunst et al., 1995). Recent studies have shown that N-terminal fragments of mutant huntingtin can form aggregates (Davies et al., 1997; DiFiglia et al., 1997; Scherzinger et al., 1997) that might play a role in cell injury.

Received April 20, 1998; revised July 15, 1998; accepted July 17, 1998.

This work was supported by a Markey Center for Neurological Sciences grant (C.-A.G.), by the Huntington's Disease Society of America and the Veterans Administration (R.J.F.), by the Hereditary Disease Foundation and the Wills Foundation and Whitehall Foundation (X.-J.L.), and by National Institutes of Health Grants NS01624 (S.M.H.), NS35255 (S.M.H., C.-A.G., and R.J.F.), and NS36232 (X.-J.L.). We thank Ryan Berglund and Shannon Mulroy for their assistance with illustrations.

Correspondence should be addressed to Dr. Steven M. Hersch, Department of Neurology, WMRB Suite 6000, Emory University School of Medicine, Atlanta, GA 30322 .

Copyright $\odot 1998$ Society for Neuroscience $0270-6474 / 98 / 187674-13 \$ 05.00 / 0$
Other protein-protein interactions may also be important in the pathogenesis of $\mathrm{HD}$. Huntingtin interacts with several proteins including huntingtin-associated protein 1 (HAP1) (Li et al., 1995, 1996), glyceraldehyde phosphate dehydrogenase (Burke et al., 1996), an unidentified calmodulin-associated protein (Bao et al., 1996), a ubiquitin-conjugating protein (HIP2) (Kalchman et al., 1996), and a protein homologous to the yeast cytoskeleton-associated protein Sla2p (huntingtininteracting protein 1 or HIP1) (Kalchman et al., 1997; Wanker et al., 1997). Binding of both HAP1 and HIP1 varies with the length of the polyglutamine tract; thus their normal function may be altered by the HD mutation.

HAP1 was first identified by yeast two-hybrid screening (Li et al., 1995). The highest levels of HAP1 are in the striatum, olfactory bulbs, and brainstem, whereas lower levels were identified in the cerebral cortex, hippocampus, and cerebellum (Li et al., 1995, 1996), regions with the highest levels of huntingtin expression ( $\mathrm{Li}$ et al., 1993; Strong et al., 1993; Landwehrmeyer et al., 1995). Despite regional differences in HAP1 and huntingtin expression, their distribution within neurons may be quite similar. Subcellular fractionation studies have shown that both HAP1 and huntingtin are present in soluble and membrane-containing fractions (S.-H. Li et al., 1993; Sharp et al., 1995; X.-J. Li et al., 1996). Two recent studies have shown that HAP-1 interacts with dynactin P150 Glued (Engelender et al., 1997; Li et al., 1998a), suggesting its possible role in intracellular transport. HAP1 may be involved in trafficking of organelles or proteins such as huntingtin. The HD mutation may alter the normal transport of huntingtin via HAP1 
and lead to abnormal accumulation and aggregation in neuronal processes.

Using pre-embedding immunogold electron microscopy, we show that HAP1 and huntingtin have very similar cellular and subcellular localizations and provide further evidence that HAP1 and huntingtin may have roles in intracellular trafficking. We also show that huntingtin-containing intranuclear and neuritic aggregates found in HD do not contain HAP1. Finally we found HAP1 to be a marker of the neuronal structures identified as stigmoid bodies.

\section{MATERIALS AND METHODS}

Antibodies. Rabbit polyclonal antibodies specific for HAP1 were raised against a recombinant protein derived from a segment of the rat HAP1 (amino acids 287-452) fused to glutathione-S-transferase (GST) and affinity purified, as described previously (Li et al., 1995). Rat monoclonal antibodies specific for huntingtin (mHD549) were raised against a segment of the human huntingtin (amino acids 549-659) fused to GST, as described previously (Gutekunst et al., 1995). To detect N-terminal huntingtin aggregates, we also used a polyconal antibody (EM48) developed against the first 253 amino acids of huntingtin ( $\mathrm{Li}$ and $\mathrm{Li}, 1998$ ). Polyclonal antibodies were used at 1:1000, and mHD549 was used at 1:100 in the following immunocytochemistry experiments. Mouse antihuman mitochondrial monoclonal antibodies MAB1273 (Chemicon, Temecula, CA) and MCA151A (Serotec, Oxford, UK) were obtained commercially and used in human brain tissue at 1:500-750.

Immunocytochemistry. Adult male Sprague Dawley rats $(n=6)$ were used for light microscopic immunocytochemistry. Each rat was deeply anesthetized with an overdose of $4 \%$ chloral hydrate, injected intraperitoneally with $300 \mathrm{IU}$ of heparin, and then perfused intracardially with $0.9 \%$ saline containing $0.005 \%$ sodium nitroprusside for $1 \mathrm{~min}$, followed by $3 \%$ paraformaldehyde in $0.1 \mathrm{M}$ phosphate buffer at $\mathrm{pH} 7.2$ (PB) for 10 min at a rate of $20 \mathrm{ml}$ per min, followed by $10 \%$ sucrose in $0.1 \mathrm{M} \mathrm{PB} \mathrm{(200}$ $\mathrm{ml}$ for $10 \mathrm{~min}$ ). Brains were removed and cryoprotected in $30 \%$ sucrose at $4^{\circ} \mathrm{C}$. Brains were sectioned at $40 \mu \mathrm{m}$ using a freezing microtome, collected in PB, and rinsed in $0.05 \mathrm{M}$ Tris-buffered saline (TBS), $\mathrm{pH}$ 7.2. Free-floating sections were preblocked in $4 \%$ normal goat serum (NGS) in TBS, $0.1 \%$ Triton $\mathrm{X}-100$, and avidin $(10 \mu \mathrm{g} / \mathrm{ml})$ for $1 \mathrm{hr}$ at $4^{\circ} \mathrm{C}$. Sections were incubated in primary antibodies in TBS containing $2 \%$ NGS, $0.1 \%$ Triton $\mathrm{X}-100$, and biotin $(50 \mu \mathrm{g} / \mathrm{ml})$ at $4^{\circ} \mathrm{C}$ for $48 \mathrm{hr}$. Sections were then rinsed in TBS for a total of $1 \mathrm{hr}$ and incubated overnight in biotinylated goat anti-rabbit antibody for HAP1 and goat anti-rat antibody (ABC Elite; Vector Laboratories, Burlingame, CA) for HD549 in TBS containing $2 \%$ NGS. After several rinses in TBS, the sections were incubated in avidin-biotin complex (ABC Elite; Vector) for $1 \mathrm{hr}$ and rinsed several times in TBS. Immunoreactivity was visualized by incubation in $0.05 \% 3,3^{\prime}$-diaminobenzidine tetrahydrochloride (DAB; Sigma, St. Louis, MO) and $0.01 \%$ hydrogen peroxide in $0.05 \mathrm{M}$ Tris buffer, $\mathrm{pH}$ 7 , until a dark brown reaction product was evident (5-10 $\mathrm{min})$. Controls included the omission of primary antisera and preabsorption of antibody with excess respective GST fusion proteins (HAP1 or huntingtin) for 1 $\mathrm{hr}$ at room temperature.

Five additional rats were used for both immunoperoxidase and preembedding immunogold electron microscopy experiments. These rats were anesthetized as described above and perfused with $200 \mathrm{ml}$ of $3 \%$ paraformaldehyde and $0.2 \%$ glutaraldehyde in $0.1 \mathrm{M} \mathrm{PB}$ at $\mathrm{pH}$ 7.2. Brains were sectioned at $50 \mu \mathrm{m}$ using a vibratome (Pelco, Redding, CA). Immunoperoxidase staining was performed as described above except that Triton X-100 was omitted from the blocking steps and from antibody solutions. After DAB visualization, sections were osmicated $\left(1 \% \mathrm{OsO}_{4}\right.$ in $0.1 \mathrm{M}$ cacodylate buffer), rinsed, and stained overnight in $2 \%$ aqueous uranyl acetate.

For more precise spatial resolution, we used pre-embedding immunogold labeling of HAP1 and huntingtin. Vibratome sections were preblocked in 5\% NGS and $0.05 \%$ Triton X-100 in TBS for 30 min and incubated at $4{ }^{\circ} \mathrm{C}$ on a shaker platform in primary antibodies in $2 \%$ NGS in TBS for $60 \mathrm{hr}$. Sections were then rinsed in six changes of TBS for a total of $60 \mathrm{~min}$ and were incubated overnight in Fab fragments of goat anti-rat secondary antibodies (1:50) conjugated to $1.4 \mathrm{~nm}$ gold particles (Nanoprobes, Stony Brook, NY) in TBS with 2\% NGS. After rinsing in TBS and then PB, sections were fixed with $2 \%$ glutaraldehyde in $0.1 \mathrm{M}$ $\mathrm{PB}$. After several washes in PB, sections were silver intensified following a modification (Yi and Hersch, 1997) of the Burry et al. (1992) method, post-fixed for $10 \mathrm{~min}$ in $0.5 \% \mathrm{OsO}_{4}$ in $\mathrm{PB}$, and processed for electron microscopy as described below.

Two additional animals were perfused with $3 \%$ paraformaldehyde and $2.0 \%$ glutaraldehyde to provide optimally fixed tissue for examining unlabeled cytoplasmic inclusions. All the sections used for electron microscopy were dehydrated in ascending concentrations of ethanol and propylene oxide (1:1) and embedded in Eponate 12 (Ted Pella, Redding, CA). Ultrathin sections $(90 \mathrm{~nm})$ were cut with a Leica Ultracut S ultramicrotome and examined with a JEOL $100 \mathrm{C}$ electron microscope. Thin sections were counterstained with 5\% aqueous uranyl acetate for 5 min, followed by Reynolds lead citrate (Reynolds, 1963) for 5 min.

To examine the normal distribution of HAP1 in the human, we performed immunocytochemistry on postmortem striatal and cortical tissue from 23 male and female humans with no evidence of neurological disease. Ages of the controls ranged from 54 to 79 years (mean age, 64.3 years). We also performed immunocytochemistry on four adult-onset grade 3 and grade $4 \mathrm{HD}$ cases to determine whether HAP1 was contained within the recently described protein aggregates containing $\mathrm{N}$-terminal fragments of huntingtin. Ages of the HD cases ranged from 42 to 78 years (mean age, 68.6 years). Intact hemispheres were placed in cold $\left(4^{\circ} \mathrm{C}\right) 2 \%$ paraformaldehyde-lysine-periodate solution overnight. Hemispheres were then sliced coronally $(1 \mathrm{~cm}$ thick) and fixed another day in fresh fixative solution. Tissue slices were rinsed in $0.1 \mathrm{~m}$ sodium phosphate buffer and placed in cold cryoprotectant in increasing concentrations of 10 and $20 \%$ glycerol and $2 \%$ DMSO solution. Blocks including the regions of interest were dissected, and frozen serial sections were cut at $50 \mu \mathrm{m}$ intervals. The cut sections were stored in $0.1 \mathrm{~m}$ sodium phosphate buffer and $0.08 \%$ sodium azide at $4^{\circ} \mathrm{C}$ for subsequent immunocytochemistry. Immunocytochemistry was performed as recently described (Ferrante et al., 1997).

Immunogold quantification. Quantification of the immunogold labeling within dendrites was performed in the frontal cortex so that longitudinally sectioned apical dendrites of pyramidal cells could be used to maximize the visualization of microtubules. A similar comparison was not performed for axon terminals because too few well-labeled huntingtin-immunoreactive terminals were identified in any brain region. For each antigen, one block each from the frontal cortex from two different brains was selected, thin sectioned, mounted on mesh grids, and stained as described previously. Three to four grid squares containing the tissue/plastic interface were scanned, and every dendritic profile containing more than five immunogold particles was photographed at $13,000 \times$. The electron micrographs were examined, and the localization and associations of each individual immunogold particle were assessed. Potential associations were with the plasma membrane, microtubules, mitochondria, and tubulovesicular elements (smooth endoplasmic reticulum and vesicles). Immunogold particles not associated with any structure were designated cytoplasmic, even though they could be in contact with structures above or below the plane of the section. When clusters of particles (usually two to four) were encountered, an effort was made to assess each particle individually. Particles were considered to be associated with an organelle if they were in direct contact. If there was any space at all between an immunogold particle and an organelle, they were not considered to be associated. Because dendritic profiles varied greatly in size and labeling density, statistical treatments in which individual profiles are treated as comparable counting objects could not be used. The association data for each antibody were summed, and the proportions of immunogold particles associated with a given organelle or compartment were calculated.

Subcellular fractionation and microtubule preparation. Subcellular fractions of rat brain were prepared essentially as described previously (Huttner et al., 1983). Tissue homogenate was centrifuged for $10 \mathrm{~min}$ at $1000 \times g$ to produce a crude nuclear fraction $(\mathrm{P} 1)$. The supernatant $(\mathrm{S} 1)$ was then centrifuged at $10,000 \times g$ for 15 min to produce a pellet $(\mathrm{P} 2)$ and a supernatant (S2). S2 was centrifuged at $100,000 \times g$ for $2 \mathrm{hr}$ to give pellet (P3) and soluble (S3) fractions. The P2 fraction was resuspended in a small volume of buffered sucrose and hypotonically lysed by addition of $10 \mathrm{vol}$ of ice-cold water on ice for $30 \mathrm{~min}$. The P2 lysate was centrifuged for $20 \mathrm{~min}$ at $25,000 \times g$ to yield the lysate pellet (LP1) and lysate supernatant (LS1). The LS1 fraction was then centrifuged at 165,000 $\times$ $g$ for $2 \mathrm{hr}$ to give a crude synaptic vesicle pellet (LP2) and supernatant (LS2). Equal amounts of protein from each fraction $(65 \mu \mathrm{g} / \mathrm{lane})$ were resolved in $4-12 \%$ SDS gels, and Western blot analysis was conducted with antibodies to HAP1 (Li et al., 1995), huntingtin (Gutekunst et al., 1995), and synaptophysin (Boehringer Mannheim, Indianapolis, IN).

Rat brain microtubule pellets were prepared as described previously 

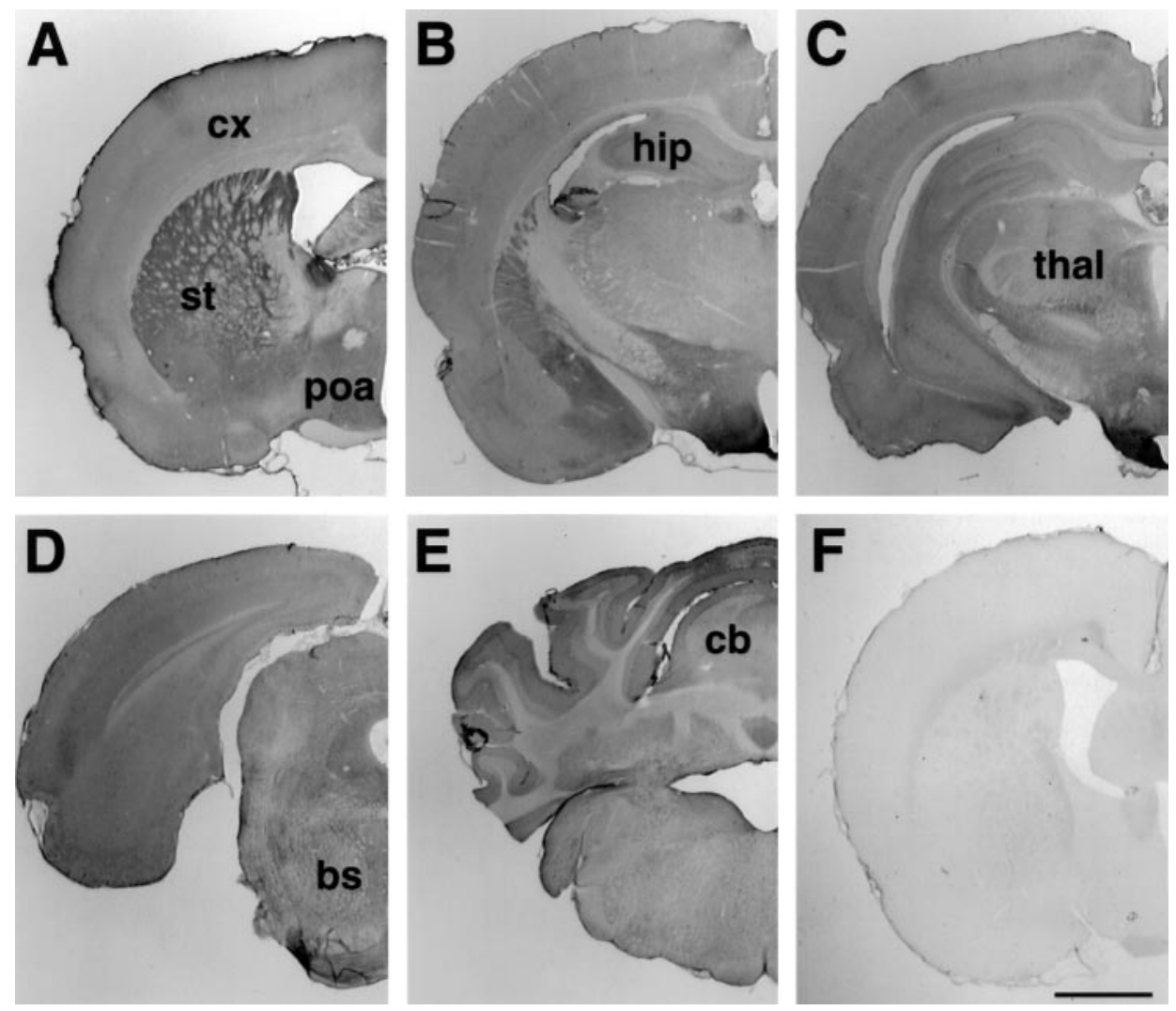

Figure 1. Regional distribution of HAP1 in the rat brain. $A-E$, Representative coronal sections from HAP1-immunostained tissue are shown from rostral to caudal. $F$, The immunolabeling is abolished after preabsorption over excess GST-HAP1 fusion protein. $b s$, Brainstem; $c b$, cerebellum; $c x$, cortex; hip: hippocampus; poa, preoptic area; $s t$, striatum thal, thalamus. Scale bar, $1 \mathrm{~mm}$.
(Vallee, 1982). Microtubules in the rat brain cytosolic extract in PIPES buffer (50 mM PIPES, $1 \mathrm{~mm}$ EGTA, and $1 \mathrm{~mm} \mathrm{MgSO}_{4}, \mathrm{pH}$ 6.9) were polymerized in the presence of $1 \mathrm{~mm}$ GTP and $20 \mathrm{~mm}$ taxol (Sigma) for $20 \mathrm{~min}$ at room temperature. Polymerized microtubles were pelleted by centrifugation at $100,000 \times g$ for $30 \mathrm{~min}$ at room temperature. The microtubules were resuspended in the same PIPES buffer and precipitated again by centrifugation through a $20 \%$ sucrose cushion. The cytosolic fraction and microtubule pellets ( $80 \mu \mathrm{g}$ of protein per lane) were subjected to Western blot analysis with antibodies to HAP1 and huntingtin.

\section{RESULTS}

\section{Cellular distribution of HAP1}

Although HAP1 immunoreactivity was widespread in the rat brain, there were regional differences (Fig. 1) with the most intense labeling being evident in the striatum, globus pallidus, amygdala, hypothalamus, and much of the ventral forebrain. Individual laminae were intensely stained in other areas, such as layer $\mathrm{V}$ of the cerebral cortex, the hippocampal pyramidal cell layer, and the Purkinje cell layer of the cerebellum. There were HAP1-immunoreactive neurons in every region of the brain examined, with minimal labeling of glia and only modest labeling of white matter tracts (Figs. 1, 2). In all cases, immunoreactivity was abolished when primary antibodies were either omitted or preabsorbed against GST-HAP1 fusion protein (Fig. $1 F$ ).

The cellular pattern of immunostaining for HAP1 was heterogeneous in most rat brain regions with both intensely labeled and lightly labeled neurons evident (Fig. $2 A-D$ ). This pattern of heterogeneity was very similar to that of huntingtin immunolabeling from contiguous sections (Fig. $2 E-H$ ). HAP1 immunocytochemistry, however, often revealed more neurons than did huntingtin immunocytochemistry. In the cerebral cortex (Fig. $2 A, E)$, layer $\mathrm{V}$ pyramidal cells and scattered layer III and VI neurons were most intensely stained by both antibodies; however, more lightly stained neurons in all layers were evident with HAP1 immunocytochemistry. Few nonpyramidal cells were evident with either antibody. In the striatum (Fig. $2 B, F$ ), populations of lightand dark-stained neurons were evident with both antibodies, but more neurons were evident with HAP1 immunocytochemistry. More medium-sized neurons and more neurons with the appearance of striatal interneurons appeared to be HAP1 immunoreactive, in comparison with huntingtin. In the globus pallidus (Fig. $2 C, G)$, neurons and dendrites were similarly well stained by HAP1 and huntingtin immunocytochemistry. In both the striatum and pallidum, the neuropil was more intensely stained by HAP1 than by huntingtin immunocytochemistry. Regional heterogeneity consistent with striosome or patch and matrix organization was not evident with HAP1 immunocytochemistry. In the hippocampus, both pyramidal and granule cells stained for both HAP1 and huntingtin. In the cerebellum (Fig. 2D, H), Purkinje cells were densely stained by both HAP1 and huntingtin antibodies, whereas the granule cell layer was relatively lightly stained. Individual HAP1-immunoreactive neurons contained reaction product primarily in their perikarya and proximal dendrites (Fig. $2 A-D)$. In the most intensely labeled neurons, however, extensive labeling of dendritic trees and proximal axon segments was evident.

\section{HAP1 labeling of cytoplasmic puncta}

By light microscopy, the HAP1 immunoreaction product was not only diff usely present in neuronal perikarya and dendrites but was also evident as intensely labeled cytoplasmic puncta (Fig. 3). These puncta were observed in neurons throughout the brain and could be divided into two types based on their size, labeling intensity, and number per neuron. The first type of puncta (Fig. 

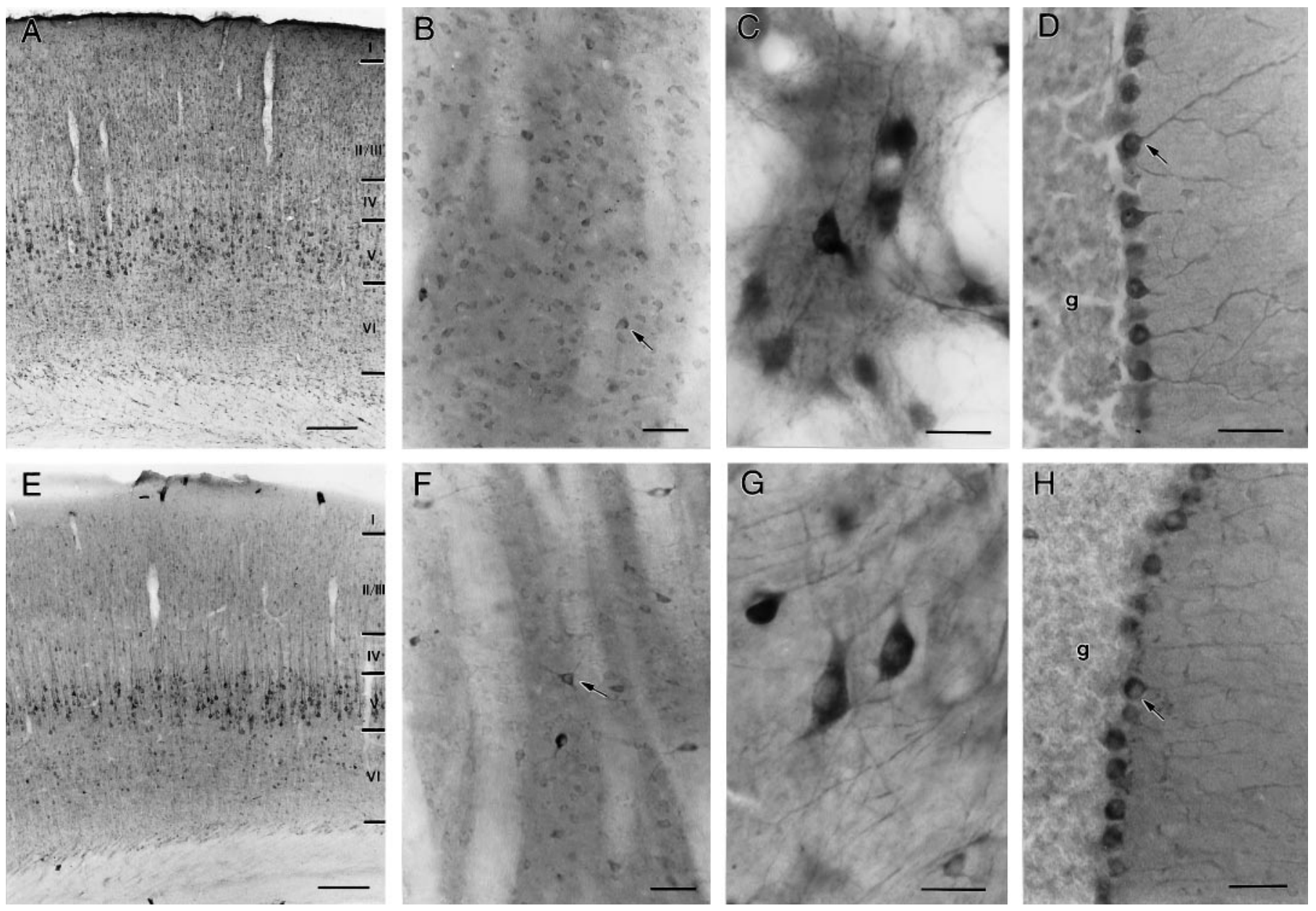

Figure 2. Similarities in HAP1 and huntingtin distribution in the rat brain. Light micrographs showing HAP1 $(A-D)$ and huntingtin Hp549 $(E-H)$ immunoreactivity are shown. Both HAP1 and huntingtin immunolabeling filled neuronal perikarya and dendrites. Throughout the brain, HAP1 cellular distribution was very similar to that of huntingtin; however HAP1 labeled more neurons than did huntingtin. $A, E$, In somatosensory cortex, HAP1 ( $A$ ) and huntingtin $(E)$ are found in pyramidal neurons. $B, F$, In striatum, both medium-sized neurons and larger neurons (arrows) are labeled with HAP1 $(B)$ and huntingtin $(F) . C, G$, In globus pallidus, neurons and dendrites are well stained by both HAP1 $(C)$ and huntingtin $(G)$. $D, H$, In the cerebellum, Purkinje cells (arrows) are more intensely stained by both HAP1 $(D)$ and huntingtin $(H)$ than are neurons in the granule cell layer $(g)$. Scale bars: $A$, $E, 200 \mu \mathrm{m} ; B, D, F, H, 50 \mu \mathrm{m} ; C, G, 25 \mu \mathrm{m}$.

$3 A-D$ ) was intensely labeled and ranged from 0.5 to $4 \mu \mathrm{m}$ in diameter. These puncta were evident in pyramidal and nonpyramidal neurons of the cerebral cortex (Fig. $3 A$ ), in medium-sized striatal neurons (Fig. 3B), and in scattered neurons in most brain regions. Neurons containing the largest and greatest numbers of these puncta were in the medial preoptic area (Fig. $3 C$ ), the lateral septum (Fig. 3D), many hypothalamic nuclei, the bed nucleus of the stria terminalis, and the medial amygdala. Less than 12 of these puncta were observed in the cytoplasm of the neurons containing them. The second type of puncta was less intensely labeled, much more frequent, and ranged from 0.5 to 1 $\mu \mathrm{m}$ in diameter. They were very numerous with $>50$ countable per neuron where they were observed in perikarya and proximal dendrites. These smaller puncta were widely distributed but were especially discernible in larger, well-stained cells such as globus pallidus neurons (Fig. $3 E$ ), pontine motor neurons (Fig. $3 F$ ), and Purkinje cells (Fig. $3 G$ ). The large and small puncta often coexisted in individual neurons (Fig. $3 F$ ). The nature of these puncta was subsequently determined using immunogold electron microscopy.

\section{HAP1 labeling in human brain}

To determine whether the punctate pattern of labeling was also present in the human, we performed HAP1 and huntingtin immunocytochemistry in sections from the putamen (Fig. $4 A, C$, respectively) and frontal cortex (Fig. $4 B, D$, respectively) of adult human postmortem brains. The visualization of inclusions in the human was aided by Nomarski optics. Both sizes of puncta could be distinguished with both HAP1 and huntingtin immunocytochemistry. The first type was large and thick-walled by Nomarski optics. Most neurons contained zero to five of these, and they ranged from $\sim 1$ to $4 \mu \mathrm{m}$ in diameter. These were considerably darker and easier to visualize in HAP1 material. The second type was smaller $(<1 \mu \mathrm{m}$ in diameter) and very numerous and was visible as hollow ovoids by Nomarski optics. This latter type of inclusion was readily observed with both HAP1 and huntingtin antibodies and was similar in size and shape to mitochondria that were labeled for comparison by the use of specific antibodies (Fig. 4E).

Recent studies have demonstrated the presence of intranuclear and neuritic protein aggregates containing the $\mathrm{N}$-terminal portion 

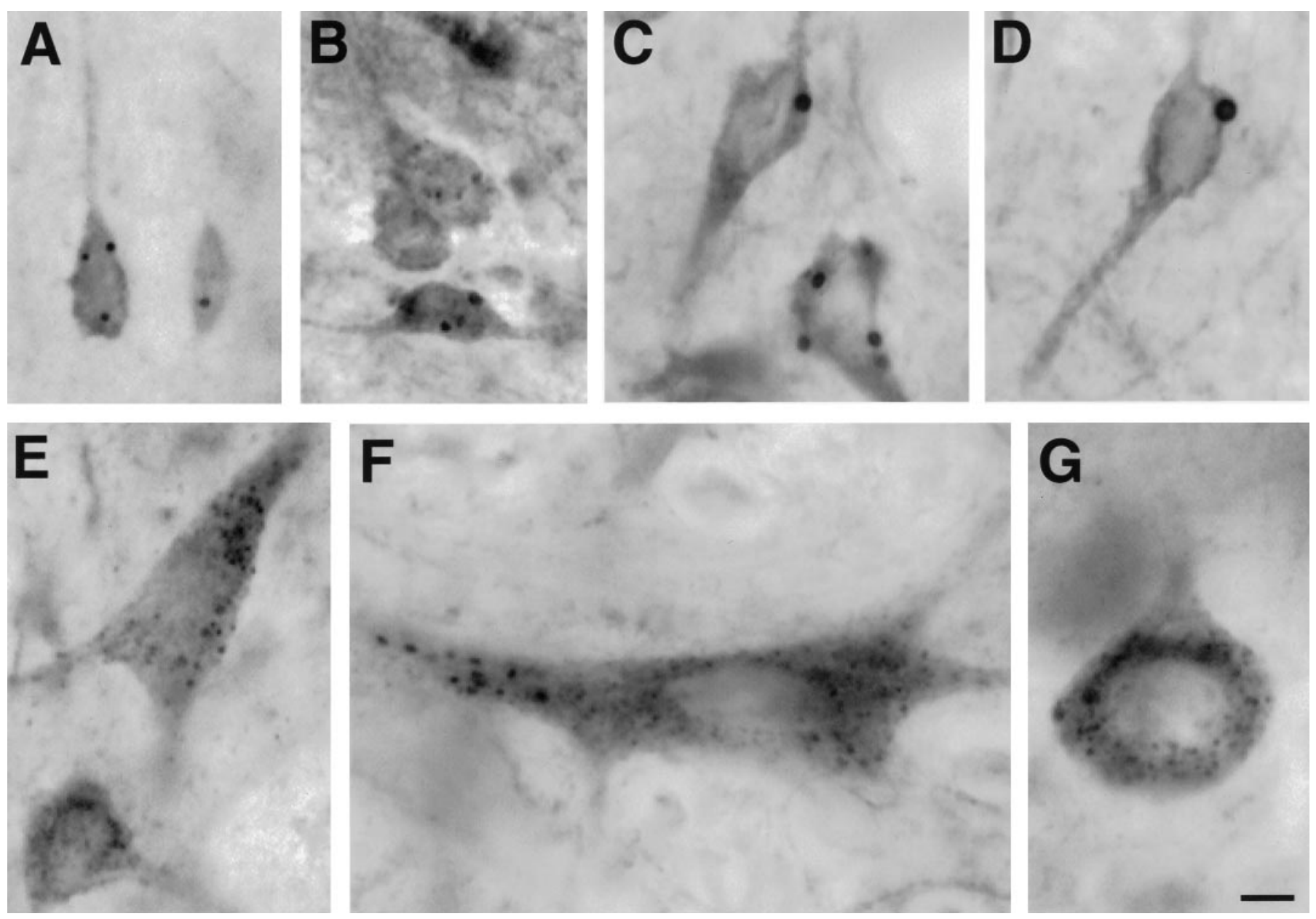

Figure 3. HAP1 labeling of large and small inclusions in the rat brain. Light micrographs showing HAP1 immunolabeling of inclusions in neurons from the cortex $(A)$, striatum $(B)$, preoptic area $(C)$, and the septal region $(D)$ are shown. In globus pallidus $(E)$, brainstem neurons $(F)$, and Purkinje cells $(G)$, HAP1 immunoperoxidase labels organelles that are smaller and more numerous than stigmoid bodies and may primarily be mitochondria. Scale bar, $10 \mu \mathrm{m}$.

of huntingtin in HD postmortem brain tissue (Becher et al., 1997; DiFiglia et al., 1997). To determine whether HAP1 was present in these aggregates, we performed HAP1 immunocytochemistry on sections from several advanced HD patients including the cortex, striatum, hippocampus, and cerebellum. We first confirmed the presence of huntingtin aggregates in these cases by performing immunocytochemistry using a fusion protein antibody specific for the N-terminal portion of huntingtin ( $\mathrm{Li}$ and $\mathrm{Li}, 1998)$. Staining with this antibody labeled many aggregates in a variety of regions in all HD cases (Fig. 5A). Staining of adjacent sections with the HAP1 antibody did not label aggregates, suggesting that huntingtin aggregates do not contain a significant level of HAP1 (Fig. $5 B)$. In HD cases, HAP1 immunoreaction product filled perikarya and proximal dendrites, with a staining pattern very similar to that seen in control cases.

\section{Subcellular localization of HAP1}

The subcellular localization of HAP1 was examined in the rat by immunoperoxidase and pre-embedding immunogold electron microscopy. The regions examined included the neostriatum, frontal cortex, lateral septum, and cerebellum. There were no major differences in HAP1 localization between each of these areas. Immunolabeling was almost exclusively found in neurons and neuronal processes. The majority of identifiable astrocyte and oligodendrocyte somata were devoid of labeling. In each region examined, DAB reaction product or immunogold particles were observed labeling neuronal somata, dendrites of all calibers, and axon terminals. Although more processes were labeled with immunoperoxidase, immunogold provided superior spatial resolution, and thus the descriptions and illustrations that follow will be from this material.

Subcellular associations of HAP1 within perikarya (Fig. 6A) were very similar to those reported previously for huntingtin. Immunogold particles were primarily cytoplasmic, often found in regions rich in polysomes. Because the immunogold particles were much larger than were individual ribosomes, it was impossible to determine whether they were directly labeled. HAP1 labeling was also present in neuronal nuclei where it associated with nuclear rods (Fig. 7A). Nuclear rod labeling was also evident in human tissue (Fig. 7B). Many immunogold particles were in contact with the outer surface of membrane-bound organelles, including rough and smooth endoplasmic reticulum, tubulovesicular profiles, and mitochondria. A type of large nonmembranebound cytoplasmic body, when observed, was especially well labeled, as described more fully below. Labeling associated with 

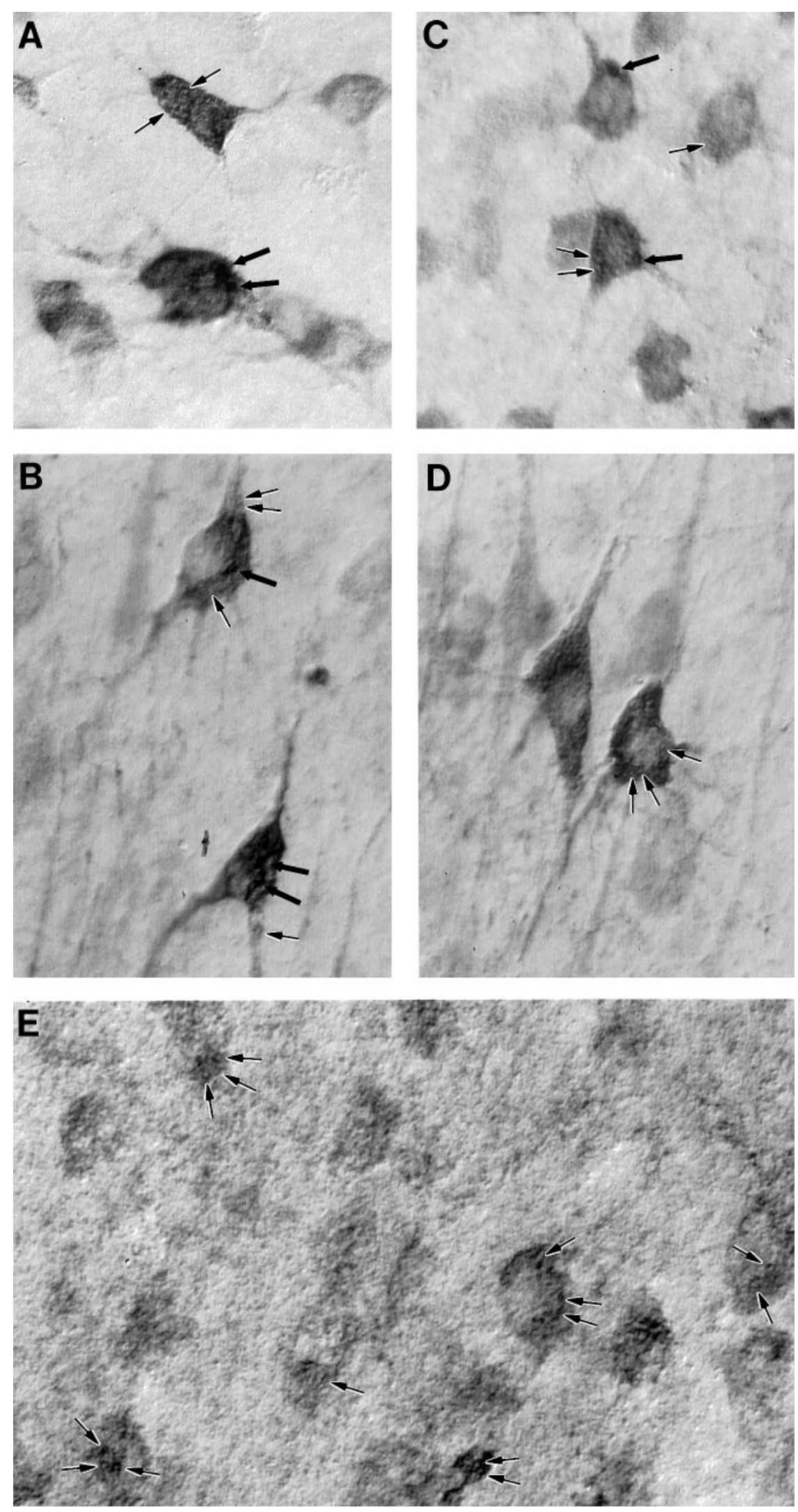

Figure 4. HAP1 immunostaining in the human and comparison with huntingtin and mitochondrial membrane markers. Nomarski imaging of HAP1 $(A, B)$ huntingtin $(C, D)$, and mitochondria $(E)$ in human brain is shown. Large and small inclusions (thick and thin arrows, respectively), similar to those visualized in the rat, are visible in these neurons from the putamen $(A, C)$ and cerebral cortex $(B, D)$. The human mitochondrial marker MAB1273 is shown $(E)$ labeling inclusions (arrows) similar in size to the small HAP1and huntingtin-immunoreactive inclusions. the Golgi apparatus was rare, as were immunogold particles in contact with the nuclear envelope or plasma membrane. Immunogold labeling of perikaryal microtubules was also evident.

Within dendrites (Fig. 6B,C), immunogold particles were rarely out of contact with organelles. Most were in contact with microtubules, mitochondria, and tubulovesicles. Rare immunogold particles were in contact with the plasma membrane, and almost none were associated with dendritic synaptic specializa- 

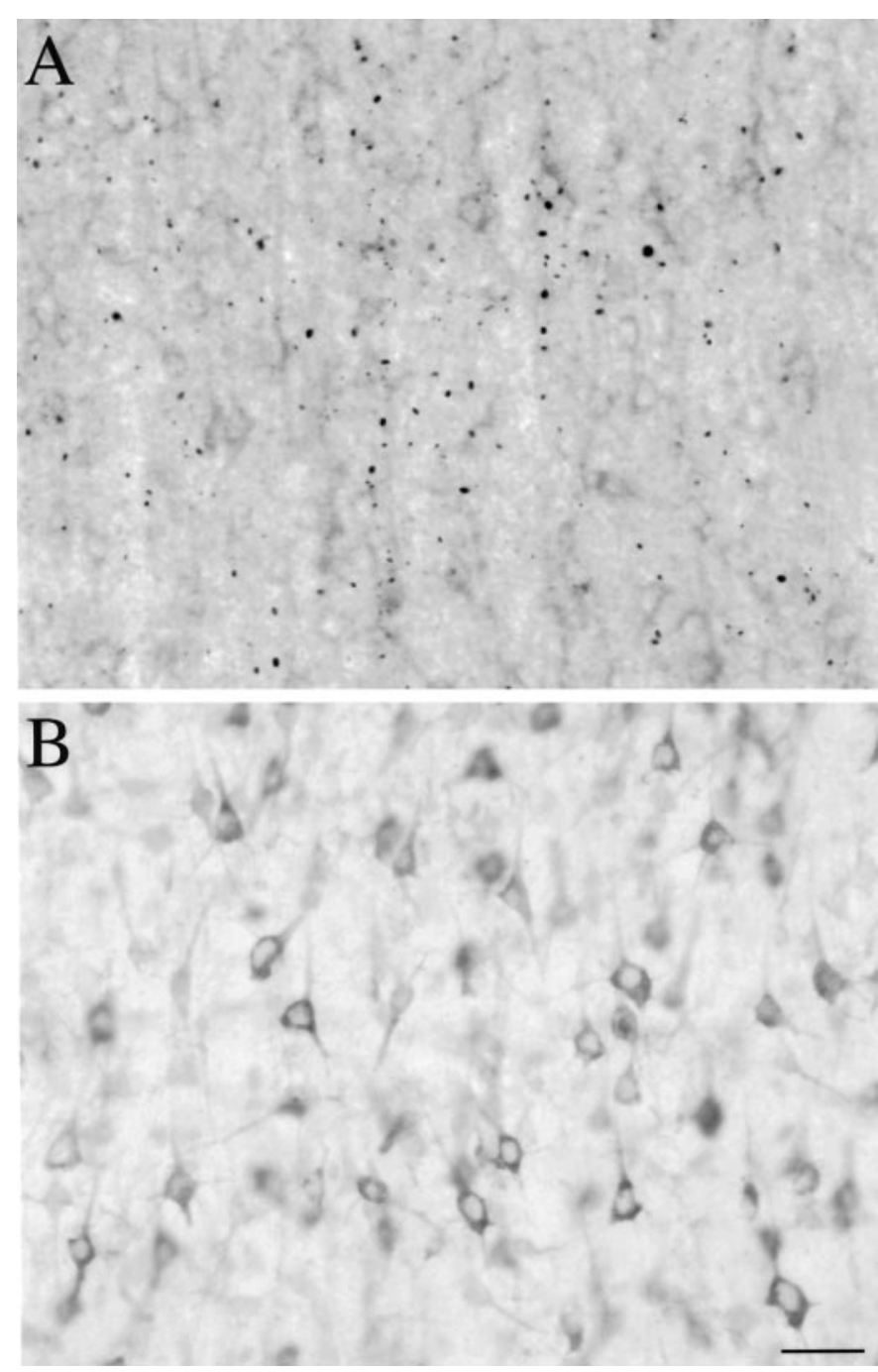

Figure 5. HAP1 is not present in N-terminal huntingtin aggregates. $A$, Light micrograph showing EM48 labeling in cortex from an HD case. Em48 immunostained many aggregates located mostly in the neuropil but also found in neuronal nuclei. $B$, An adjacent section immunolabeled with HAP1. Immunostaining is localized to neuronal perikarya and proximal processes and is diffuse in the neuropil. There is no labeling of aggregates. Scale bar, $20 \mu \mathrm{m}$.

tions. Immunogold particles observed in dendritic spines (Fig. $8 A$ ) were cytoplasmic, although sometimes associated with the spine apparatus. Axon terminals (Fig. $8 A, B$ ) were frequently labeled with immunogold particles, most of which were observed on the outer surface of synaptic vesicles. The external surface of mitochondria were also labeled in axon terminals. The plasma membranes and presynaptic densities of axon terminals were not labeled. Some myelinated axons (Fig. 8C) in the examined neuropils also contained immunogold particles that were usually associated with the axonal cytoskeleton or with mitochondria.

Sections from control experiments in which the primary antibody was omitted were examined to assess the extent of background immunogold labeling. In these sections, immunogold particles were often present, scattered throughout the tissue. When present, these occurred in any ultrastructural compartment. These were at a very low density, however, with most cells and processes being completely unlabeled. When labeled, axon terminal and dendrite profiles contained no more than two particles. These immunogold particles did not occur in clusters and did not show the selectivity described above for the surfaces of microtubules or organelles.

\section{Quantitative comparison of HAP1 and huntingtin immunogold labeling}

The subcellular associations of HAP1 with membranous organelles and microtubules are very similar to what we have reported for huntingtin (Gutekunst et al., 1995). The major qualitative differences were that HAP1 immunogold particles were more readily found in axon terminals and much more densely labeled a certain type of cytoplasmic inclusion. To provide a more quantitative comparison of these associations, we made blinded counts of all the immunogold particles visible in electron micrographs of dendrites from the cerebral cortex labeled for either HAP1 ( $n=22$ dendrites containing 276 immunogold particles) or huntingtin ( $n=20$ dendrites containing 197 immunogold particles). Because the labeling of one organelle is not really comparable with the labeling of another because of differences in surface area and frequency, this analysis does not enable the determination of the relative labeling of different organelle populations. However, it does permit fairly sensitive comparisons of the extent to which the two markers label a given organelle. The results are remarkably similar for the two markers (Fig. 9), indicating their subcellular associations to be similarly distributed within dendrites.

\section{Electron microscopic analysis of HAP1-immunoreactive puncta}

The large intracellular puncta observed by light microscopy to be densely HAP1 immunoreactive were examined using electron microscopy (Fig. 10). Because they were often so densely labeled that their substructure was obscured by immunogold particles, we also examined comparable structures in material not labeled by immunocytochemistry (Fig. $10 D, E$ ). These puncta appear identical to a group of nonmembrane-bound cytoplasmic organelles that have been identified in brain and peripheral tissues and variously termed "nucleolus-like body" (Navarro et al., 1995), "nematosomes" (Hindelang-Gertner et al., 1974), "basophilic intracellular granules" (Hindelang-Gertner et al., 1974; Holmgren et al., 1987), "stigmoid bodies" (Shinoda et al., 1993), and "botrysomes" (Kind et al., 1997). Based on the classification proposed by Shinoda et al. (1993), we will use the term stigmoid bodies. HAP1-immunoreactive stigmoid bodies were found to be heterogeneous electron-dense organelles that were ovoid to circular in shape, 0.5 to $4 \mu \mathrm{m}$ in diameter, and not enclosed by a limiting membrane. The largest ones were most easily found in perikarya (Fig. 10A). Rough endoplasmic reticulum and polyribosomes were often observed in the immediate vicinity of HAP1immunoreactive stigmoid bodies. Smaller stigmoid bodies were also observed in large to medium caliber dendrites (Fig. 10E) and, in rare instances, in axon terminals (Fig. 10F). The ultrastructure of stigmoid bodies consists of closely packed collections of electron-dense particles and fibrils frequently surrounding a central or eccentric electron-lucent core (Fig. 10B-D). Many of the individual elements making up the electron-dense shell were circular, semicircular, or tubular elements $\sim 20-50 \mathrm{~nm}$ in diameter surrounding their own electron-lucent core. Because these elements were only rarely elongated, we interpret them to repre- 

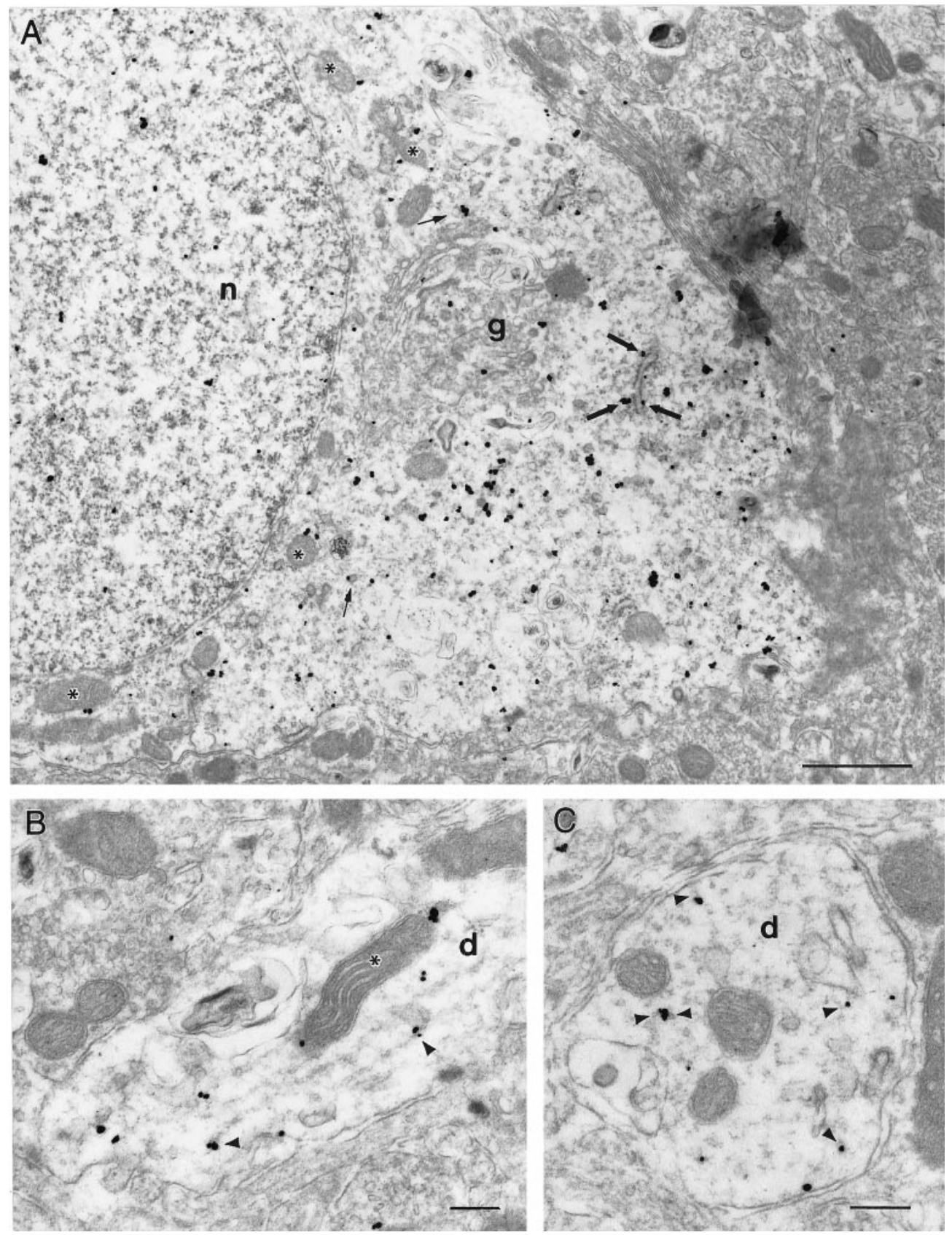

Figure 6. Subcellular localization of HAP1. $A$, Electron micrograph showing the cell body of a HAP1-immunolabeled neuron in the septal region. Most immunogold particles were cytoplasmic, whereas a few were also found in the nucleus $(n)$. Most particles were either free in the cytoplasm in regions rich in polysomes or in contact with the outer surface of rough endoplasmic reticulum (thick arrows), mitochondria (asterisks), and tubulovesicular elements (thin arrows). A few rare particles were found in contact with the plasma membrane or within regions rich in Golgi apparatus (g). B, Longitudinal section through a dendrite $(d)$ showing immunogold particles in contact with microtubules (arrowheads) or a mitochondrion (asterisk). $C$, Cross-section through a dendrite $(d)$ in which immunogold particles can be seen in contact with microtubule crosssections (arrowheads). Scale bars, 250 $\mathrm{nm}$. sent spherical collections of protein or other molecules. These elements did not appear to be vesicular because we could not discern them to have trilaminar membrane structure. Other smaller particles were similar in size and density to ribosomes. HAP1 immunogold particles most densely labeled the periphery of the stigmoid bodies (Fig. $10 A-C$ ). Immunogold particles were also found throughout the electron-dense shell but only rarely in the electron-lucent centers. The content of the electron-lucent centers was similar to that of the cytoplasm (Fig. 10B,C) and contained occasional tubulovesicles, fibrils, larger dense particles, and small particles that could be ribosomes. Unlabeled stigmoid bodies were not observed in the immunolabeled tissue.

The population of smaller HAP1 puncta seen by light microscopy appears to be made up of several different types of organelles, all of which are membrane bound. Most of these were mitochondria (see Fig. 5) that were frequently labeled with im- munogold particles, although not nearly as densely as were stigmoid bodies. Although a single section through a mitochondrion may only show labeling by a few immunogold particles, the dozens of such particles that would cover an entire mitochondrion make them more visible by light microscopy. Other types of labeled organelles with the size and shape of the small inclusions were less frequent and included multivesicular bodies and lysosomes.

\section{Subcellular fractionation}

To investigate further the apparent associations of HAP1 with microtubules and organelles, as well as that of huntingtin, we examined their subcellular fractionation in rat brain and examined whether HAP1 and huntingtin are enriched in purified microtubule fractions. Fractionation results for HAP1 and huntingtin were the same (Fig. 11A). HAP1 cloning has revealed two isoforms (HAP1A and HAP1B) resulting from alternative splic- 
Figure 7. Association of HAP1 to nuclear rods. $A$, Electron micrograph showing HAP1 immunogold in the nucleus $(n)$ of a preoptic area neuron. Gold particles are located along an intranuclear rod (arrow). The cytoplasm $(C)$ is separated from the nucleus by the nuclear envelope. $B$, Light micrograph of a coronal section through normal human cortex showing HAP1 immunostaining. In pyramidal neurons, the $\mathrm{DAB}$ reaction product is present in perikarya and proximal dendrites but is also found along intranuclear rods (arrows). Scale bars: $A, 300 \mathrm{~nm} ; B, 10 \mu \mathrm{m}$.

Figure 8. Subcellular localization of HAP1. $A, B$, Electron micrographs showing HAP1 immunogold particles in four axon terminals $(a)$ and in three of their postsynaptic dendritic spines $(s)$. In the axon terminals, immunogold particles are located amid the synaptic vesicles, frequently contacting their cytoplasmic surfaces (arrowheads) but also contacting the outer membrane of mitochondria (asterisk). Immunogold particles in dendritic spines were free in the cytoplasm. In both axon terminals and dendritic spines, immunogold particles were not found in association with synaptic junctional complexes. $C$, Electron micrograph of a myelinated axon $(a)$ showing a few immunogold particles either amid cytoskeletal elements or in contact with a mitochondrion (asterisk). Scale bars, $250 \mathrm{~nm}$.
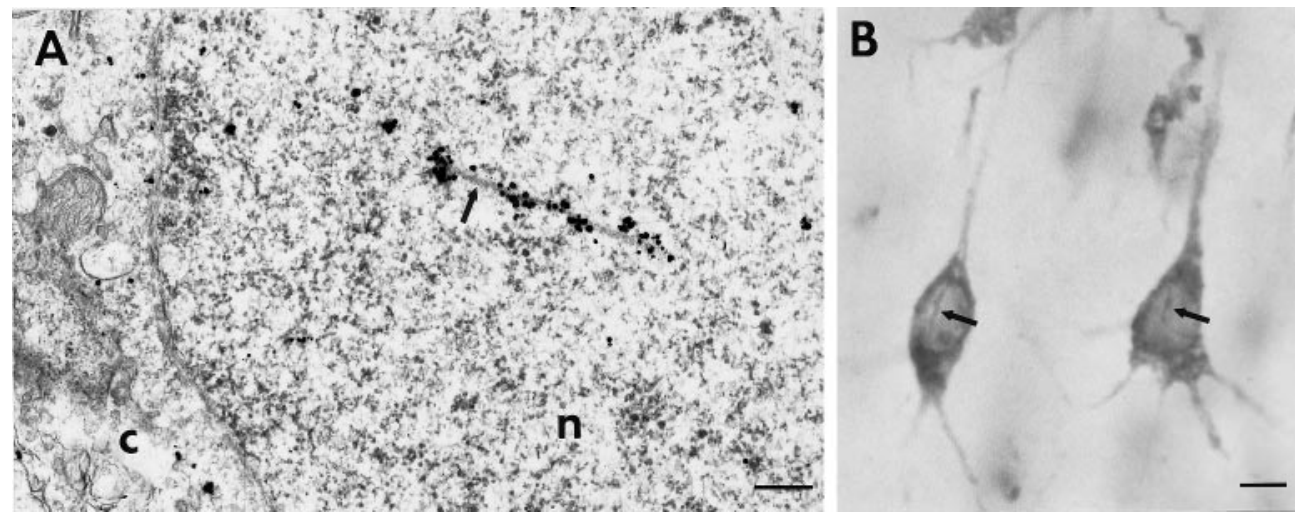
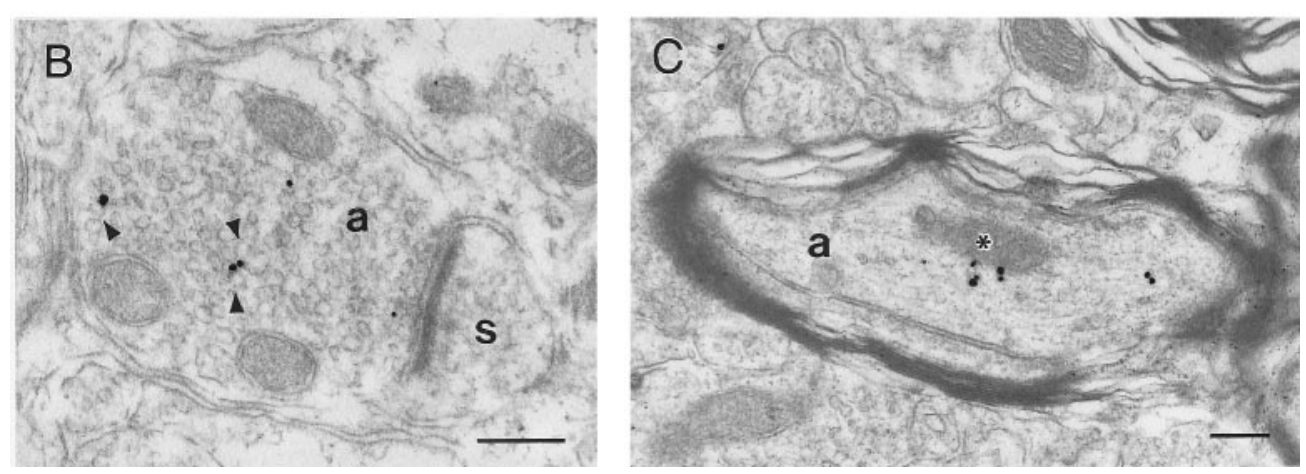

ing at the C-terminal region ( $\mathrm{Li}$ et al., 1995). The two HAP1 isoforms and huntingtin were present in membranous (P2 and P3) and cytosolic fractions (S3). Subfractionation of P2 using a hypotonic lysis method (Huttner et al., 1983) resulted in an LP2 fraction enriched in synaptic vesicles. HAP1 and huntingtin were present in the LP2 fraction along with the synaptic vesicle protein synaptophysin. After purification of microtubules (Fig. 11B) from rat brain cytosolic fractions (S3), the HAP1 isoforms remained highly enriched. In contrast, huntingtin was not as well retained in the purified microtubule fraction. 


\section{Immunogold Particle Associations in Dendrites}

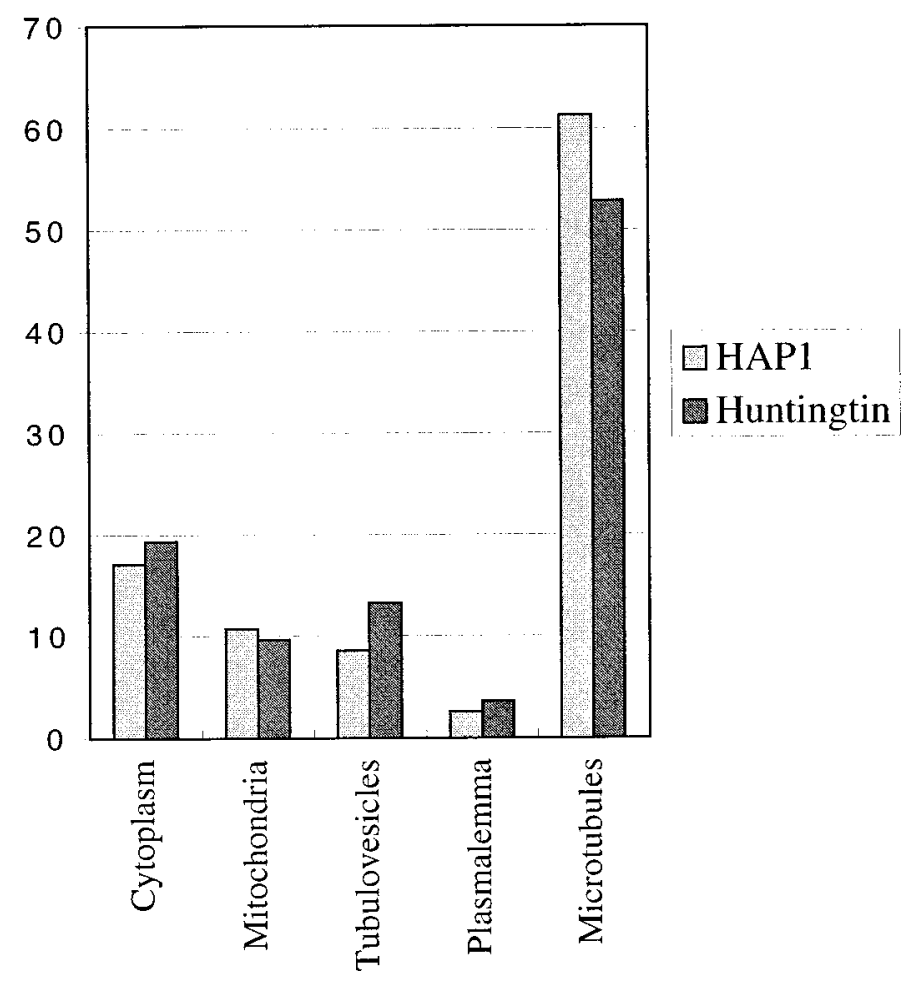

Figure 9. Comparison of the proportional labeling of organelles and membranes by HAP1 and huntingtin immunogold particles. Histograms showing the proportions of immunogold particles observed to be associated with particular subcellular compartments in frontal cortex dendrites are shown. These data indicate that the proportional distribution of HAP1 and huntingtin among the compartments is very similar. Because the frequency and surface areas of each compartment are not comparable, however, these data should not be interpreted as giving any indication of the relative abundance of HAP1 or huntingtin in each.

\section{DISCUSSION}

Our results include several major findings concerning the cellular and subcellular localization of HAP1. First, in the rat brain HAP1 is heterogeneously expressed in neurons in a pattern similar to but more widespread than that of huntingtin. Second, light and electron microscopy analyses show that HAP1 is mostly localized in the cytoplasm but is also found in nuclei where it specifically associates with nuclear rods. In the cytoplasm it strongly associates with distinct populations of large and small puncta. Although these two populations of puncta are found in human brains, they are distinct from the intranuclear and cytoplasmic protein aggregates containing the N-terminal portion of huntingtin that have been described in HD brain tissue. In fact HAP1 was not found to stain huntingtin-containing aggregates in HD tissue. At the subcellular level, HAP1 associates with microtubules and most types of membrane-bound organelles including mitochondria, endoplasmic reticulum, tubulovesicles, lysosomes, and synaptic vesicles. We also found HAP1 to be a specific marker for large nonmembrane-bound cytoplasmic bodies, recently named stigmoid bodies. The subcellular localization was confirmed by fractionation studies and is consistent with a role for HAP1 in intracellular transport. Furthermore, quantification in cortical dendrites revealed a distribution of organelle associations remarkably similar to that of huntingtin. These results show that there is a great correspondence between the subcellular distribution of HAP1 and huntingtin in rat and normal human brains, further confirming a potential functional association between these two proteins.

\section{The cellular localization of HAP1}

Our immunocytochemistry confirms earlier findings that HAP1 is selectively expressed in neurons with little or no labeling of glia. HAP1 staining was also more intense in striatum, brainstem, and ventral forebrain than in cerebral cortex and cerebellum. This is dissimilar to huntingtin immunoreactivity in which the cerebral cortex and cerebellum are the most intensely stained regions and the striatum is only moderately stained (DiFiglia et al., 1995; Gutekunst et al., 1995; Sharp et al., 1995; Trottier et al., 1995). Within a given brain region, however, the cellular patterns of HAP1 and huntingtin immunostaining were the same or very similar. With both markers, the most intensely stained neurons in the cerebral cortex were layer V and scattered layer VI pyramidal cells. Furthermore, both markers heterogeneously label populations of medium-sized striatal neurons. The differences in both these regions are that more neurons appear to be HAP1 immunoreactive than are huntingtin immunoreactive. Thus, it seems likely that huntingtin-immunoreactive neurons are a subset of HAP1-immunoreactive neurons. For example, nitric oxide synthase-containing neurons are HAP1 immunoreactive (Li et al., 1996) but appear to be devoid of huntingtin (Ferrante et al., 1997; Kosinski et al., 1997). Such differences suggest that HAP1 may have a function that does not always require the presence of huntingtin. In other regions, such as the cerebellum and globus pallidus, no differences in the cellular pattern of HAP1 and huntingtin immunoreactivity were discernible.

\section{The subcellular localization of HAP1}

The main focus of this study has been the subcellular localization of HAP1 within individual neurons as revealed by pre-embedding immunogold and subcellular fractionation. The most prominent associations found for HAP1 were with microtubules, a variety of membrane-bound organelles, and nonmembrane-bound organelles recently named stigmoid bodies. Associations with membrane-bound organelles were supported by subcellular fractionation findings in which HAP1 was found to be enriched in membrane- and synaptic vesicle-containing fractions. Because immunogold electron microscopy did not show HAP1 to be associated with nuclear and plasma membranes, the fractionated membranes must originate from organelles. Similarly, copurification with microtubules indicates that the HAP1/microtubule association observed by EM is specific. Immunogold localization of the microtubule-based transport protein kinesin (Hirokawa et al., 1991) and of MAP1 and MAP2 (Leterrier et al., 1994) has shown similar labeling of microtubules and membrane-bound organelles. By the use of the same pre-embedding immunogold technique to study the subcellular localization of cytoplasmic proteins and mRNA, neither microtubules nor membrane-bound organelles were labeled (Kachidian and Pickel, 1993; Trembleau et al., 1994; Feng et al., 1997). The observed associations with particular membrane-bound organelles also seem to be specific because not all cellular membranes are labeled (the nuclear membrane, Golgi apparatus, and plasmalemma were labeled at background levels). We thus believe that these localizations for HAP1 are specific and suggest a role for HAP1 in the microtubule-based localization or transport of organelles. We recently reported that HAP1 specif- 

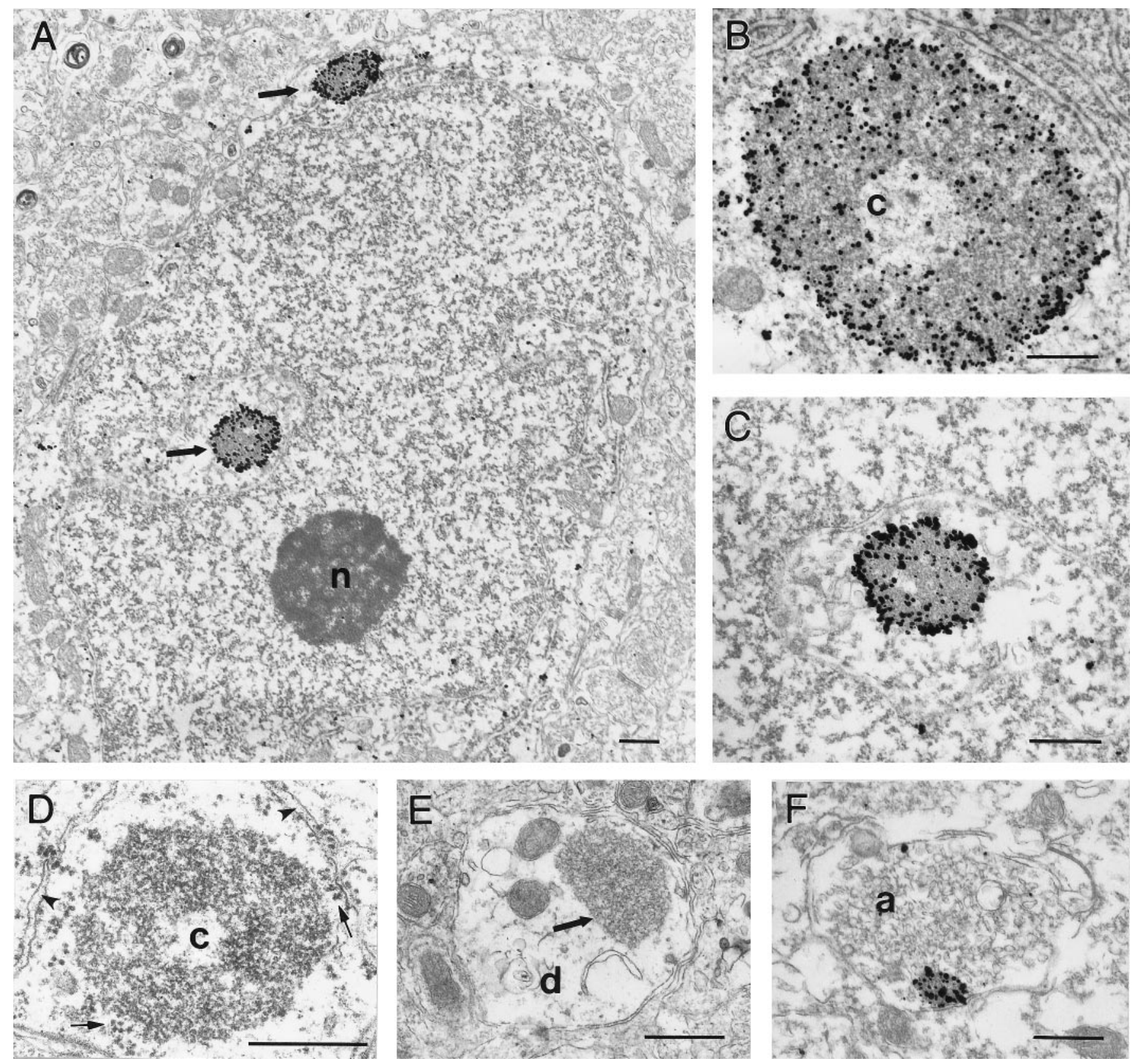

Figure 10. Labeled and unlabeled stigmoid bodies in neurons. $A-F$, Electron micrographs of stigmoid bodies demonstrating their structure, location, and HAP1 immunoreactivity. Stigmoid bodies do not have a limiting membrane and are made up of heterogeneous electron-dense material, often surrounding a large electron-lucent core $(c)$. Much of the dense material appears to be made up of individual rounded electron-dense elements with electron-lucent centers. $A$, Electron micrograph showing two immunolabeled stigmoid bodies (arrows) in the perikaryon of a neuron in the lateral septum. This perinuclear positioning is very frequent. Immunogold particles are visible both around and within the stigmoid bodies. $B, C$, Immunogoldlabeled stigmoid bodies in neurons from the preoptic area and the lateral septum seen at higher magnification. Label is concentrated on the surface of the stigmoid bodies but also is found deep within the electron-dense material. $D$, Electron micrograph of unlabeled stigmoid bodies in the perikaryon of a preoptic neuron showing stigmoid ultrastructure unobscured by immunogold particles. $E, F$, Unlabeled stigmoid (arrow) in a medium caliber dendrite $(d)$ and a labeled stigmoid in an axon terminal $(a)$ demonstrating that they can be found in more distal locations. Scale bars, $500 \mathrm{~nm}$.

ically interacts with the accessory motor protein dynactin P150 Glued (Li et al., 1998a). These interactions are further evidence that HAP1 may play a role in intracellular transport.

\section{HAP1 is a marker for stigmoid bodies}

The ultrastructural features of the large cytoplasmic puncta labeled with the HAP1 antibodies were identical to those of a subtype of nucleolus-like bodies that have been named stigmoid bodies (Shinoda et al., 1993). Further evidence that the large
HAP1-immunoreactive puncta are identical to stigmoid bodies is that the brain regions in which they are most abundant are the same regions in which stigmoid bodies have also been reported to be concentrated (Shinoda et al., 1992). Although their function is not yet well established, the work of several groups suggested a potential role of stigmoid bodies in protein synthesis (Weakley, 1969; Kishi, 1972; Takeuchi and Takeuchi, 1982). Given the remarkable density of HAP1 labeling observed in stigmoid bodies, HAP1 may serve an important role in transporting the mo- 
$\mathbf{A}$

Figure 11. Subcellular distribution of HAP1 and huntingtin. $A$, Subcellular fractions from rat whole brain were prepared according to the method of Huttner et al. (1983). Western blot analysis was conducted with antibodies for HAP1, huntingtin, and synaptophysin. $B$, Coomassie blue staining and Western blot analysis of the rat brain microtubule $(M T)$ fraction that was obtained by polymerization of tubulin in the cytosolic fraction (S3) are shown. Western blot analysis was conducted with antibodies to HAP1 and huntingtin.

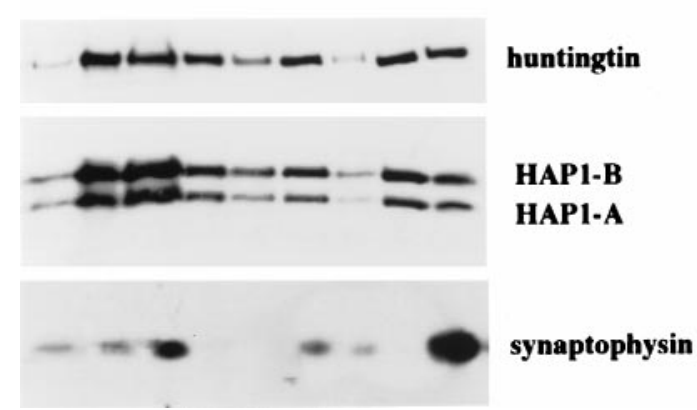

B

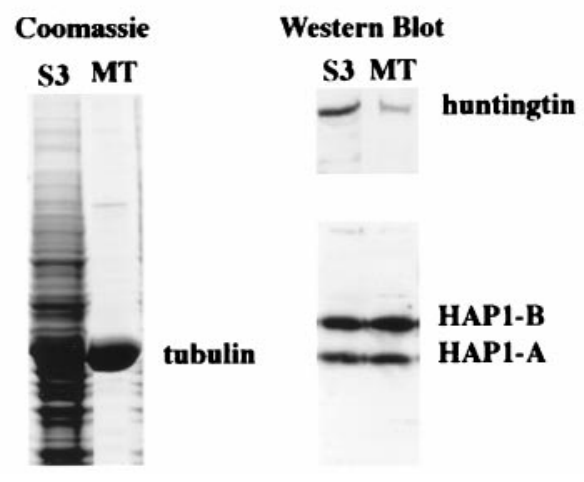

lecular constituents or products of these bodies. This possibility is supported by the finding that transfection of HAP1 cDNA into HEK293 cells results in the de novo formation of HAP1immunoreactive inclusions with a very similar ultrastructure to that of stigmoid bodies (Li et al., 1998a,b). Alternatively, HAP1 may be synthesized or stored in stigmoid bodies. Because high levels of HAP1 are found in stigmoid bodies and the binding of huntingtin to HAP1 is enhanced by the polyglutamine repeat expansion, stigmoid bodies might have a role in HD pathology. This seems unlikely, however, because stigmoid bodies are not huntingtin immunoreactive in control and HD brain and because they are most prominent in brain regions with limited HD neuropathology.

\section{HAP1 and huntingtin}

The subcellular associations of HAP1 were very similar to those we and others have reported for huntingtin and were consistent with the identified associations of these two proteins (Li et al., 1995, 1996). We have further substantiated their similarities by quantifying the relative proportions of HAP1 and huntingtin immunogold particles found to associate with particular organelles. The relative proportions of immunogold labeling of different organelles with each antibody were extremely similar, strongly supporting their association. Because the numbers and surface areas of each type of organelle are not comparable, this analysis does not address the relative density of immunogold labeling of the different organelles. For example, although microtubules were the most labeled organelle in dendrites, they also are the most numerous and have the greatest surface area. Several of the associations are quite interesting, however. Both HAP1 and huntingtin significantly labeled mitochondria. Quantification of immunogold labeling brought out the huntingtin labeling of mitochondria that had not been reported previously (DiFiglia et al., 1995; Gutekunst et al., 1995). Presumably, this association was overlooked because the nonquantitative methods used allowed the $10 \%$ association we report here to be missed. Given that the total mitochondrial surface area is relatively small, this $10 \%$ likely reflects a significant association. The potential importance of this finding relates to the relevance of mitochondria to oxidative injury, excitotoxicity, and apoptosis as mechanisms of neuronal death in HD. Perhaps HAP1 and/or huntingtin play roles in the transport, anchoring, or even turnover of mitochondria or mitochondrial constituents. Similarly, the association of HAP1 and huntingtin with synaptic vesicles suggests possible roles in vesicular trafficking. Although little is known about the transport of proteins and protein complexes, an additional possibility is that
HAP1 serves as a carrier for a heteromultimer containing huntingtin and the other proteins with which it associates. Thus, HAP1 could also be involved in the assembling or transporting of macromolecular complexes.

We have not detected HAP1 within the abnormal nuclear and cytoplasmic protein aggregates formed by the N-terminal fragments of huntingtin in HD cases. Lack of HAP1 within intranuclear protein aggregates could reflect its limited nuclear localization. Lack of HAP1 within cytoplasmic protein aggregates was more surprising because HAP1 binds more tightly to huntingtin with an expanded polyglutamine repeat than to normal huntingtin (Li et al., 1995) and the cytoplasmic subcellular localizations of both proteins are otherwise so similar in normal tissue. Although it is possible that our antibody does not penetrate the aggregate, this seems unlikely because other antibodies are able to penetrate them and because HAP1 immunoreactivity was not even present at the periphery of the aggregates. Another possibility is that HAP1 antigenicity may be altered by its binding to huntingtin fragments within the aggregates. At least in transfected cells, however, HAP1 and an N-terminal huntingtin fragment can be colocalized using the same HAP1 antibody, suggesting that binding does not interfere with antibody recognition. Finally, and most likely, there is no HAP1 protein in the protein aggregates. HAP1 may be unable to bind to aggregated huntingtin; perhaps aggregation blocks the HAP1 binding site that is close to the polyglutamine tract ( $\mathrm{Li}$ et al., 1995). It remains possible, however, that HAP1 interacts with and transports $\mathrm{N}$-terminal fragments of Huntingtin that have not yet aggregated and may even deliver them to forming aggregates.

\section{REFERENCES}

Bao J, Sharp A, Wagster M, Becher M, Schilling G, Ross C, Dawson V, Dawson T (1996) Expansion of polyglutamine repeat in huntingtin leads to abnormal protein interactions involving calmodulin. Proc Natl Acad Sci USA 93:5037-5042.

Becher MW, Kotzuk JA, Sharp AH, Davies SW, Bates GP, Price DL, Ross CA (1998) Intranuclear neuronal inclusions in Huntington's disease and dentatorubral and Pallidoluysian atrophy: correlation between the density of inclusions and IT15 CAG triplet repeat length. Neurobiol Dis 4:387-397.

Burke J, Enghild J, Martin M, Jou Y-S, Myers R, Roses A, Vance J, Strittmatter W (1996) Huntingtin and DRPLA proteins selectively interact with the enzyme GAPDH. Nat Med 2:347-350.

Burry R, Vandre D, Hayes D (1992) Silver enhancement of gold antibody probes in pre-embedding electron microscopic immunocytochemistry. J Histochem Cytochem 40:1849-1856.

Davies SW, Turmaine M, Cozens BA, DiFiglia M, Sharp AH, Ross CA, Scherzinger E, Wanker EE, Mangiarini L, Bates GP (1997) Formation 
of neuronal intranuclear inclusions underlies the neurological dysfunction in mice transgenic for the HD mutation. Cell 90:537-548.

DiFiglia M, Sapp E, Chase K, Schwarz C, Meloni A, Young C, Martin E, Vonsattel J-P, Carraway R, Reeves S, Boyce F, Aronin N (1995) Huntingtin is a cytoplasmic protein associated with vesicles in human and rat brain neurons. Neuron 14:1075-1081.

DiFiglia M, Sapp E, Chase KO, Davies SW, Bates GP, Vonsattel JP, Aronin N (1997) Aggregation of huntingtin in neuronal intranuclear inclusions and dystrophic neurites in brain. Science 277:1990-1993.

Engelender S, Sharp AH, Colomer V, Tokito MK, Lanahan A, Worley P, Holzbaur ELF, Ross CA (1997) Huntingtin-associated protein 1 (HAP1) interacts with the p150Glued subunit of dynactin. Hum Mol Genet 6:2205-2212.

Feng Y, Gutekunst C-A, Eberhart D, Yi H, Warren S, Hersch SM (1997) Fragile $\mathrm{X}$ mental retardation protein: nucleocytoplasmic shuttling and association with somatodendritic ribosomes. J Neurosci 17:1539-1547.

Ferrante R, Gutekunst C-A, Persichetti F, McNeil S, Kowall N, Gusella J, MacDonald M, Beal M, Hersch S (1997) Heterogeneous topographic and cellular distribution of huntingtin expression in the normal human neostriatum. J Neurosci 17:3052-3063.

Gutekunst C-A, Levey A, Heilman C, Whaley W, Yi H, Nash N, Rees H, Madden J, Hersch S (1995) Localization of huntingtin in rat, monkey and human tissues with anti-fusion protein antibodies. Proc Natl Acad Sci USA 92:8710-8714.

Hersch SH, Ferrante RJ (1997) Neuropathology and pathophysiology of Huntington's disease. In: Movement disorders. Neurologic principles and practice (Watts RL, Koller WC, eds), pp 503-526. New York: McGraw-Hill.

Hindelang-Gertner C, Stoeckel M-E, Porte A, Dellman H-D, Madarasz B (1974) Nematosomes or nucleolus-like bodies in hypothalamic neurons, the subfornical organ and adenohypophysial cells of the rat. Cell Tissue Res 155:211-219.

Hirokawa N, Sato-Yoshitake R, Kobayashi N, Pfister KK, Bloom GS, Brady ST (1991) Kinesin associates with anterogradely transported membranous organelles in vivo. J Cell Biol 114:295-302.

Holmgren G, Almqvist EW, Anvret M, Conneally M, Hobbs W, Mattsson B, Wahlstrom J, Winblad B, Gusella JF (1987) Linkage of G8 (D4S10) in two Swedish families with Huntington's disease. Clin Genet 32:289-294.

Huttner W, Schiebler W, Greengard P, De Camilli P (1983) Synapsin I (protein I), a nerve terminal-specific phosphoprotein. III. Its association with synaptic vesicles studied in a highly purified synaptic vesicle preparation. J Cell Biol 96:1374-1388.

Kachidian P, Pickel V (1993) Localization of tyrosine hydroxylase in neuronal targets and efferents of the area postrema in the nucleus tractus solitarius of the rat. J Comp Neurol 329:337-353.

Kalchman M, Koide H, McCutcheon K, Graham R, Nichol K, Nishiyama K, Kazemi-Esfarjani P, Lynn F, Wellington C, Metzler M, Goldberg Y, Kanazawa I, Gietz R, Hayden M (1997) HIP1, a human homolog of $S$. cerevisiae Sla2p, interacts with membrane associated huntingtin in the brain. Nat Genet 16:44-53.

Kalchman MA, Graham RK, Xia G, Koide HB, Hodgson JG, Graham KC, Goldberg YP, Gietz RD, Pickart CM, Hayden MR (1996) Huntingtin is ubiquitinated and interacts with a specific ubiquitinconjugating enzyme. J Biol Chem 271:19385-19394.

Kind P, Kelly G, Fryer H, Blakemore C, Hockfield S (1997) Phospholipase $\mathrm{C}-\beta 1$ is present in the botrysome, an intermediate compartmentlike organelle, and is regulated by visual experience in cat visual cortex. J Neurosci 17:1471-1480.

Kishi K (1972) Fine structural and cytochemical observations on cytoplasmic nucleolus-like bodies in nerve cells of rat medulla oblongata. Z Zellforsch Mikrosk Anat 132:523-532.

Kosinski C, Cha J-H, Young A, Persichetti F, MacDonald M, Gusella J, Penney Jr J, Standaert D (1997) Huntingtin immunoreactivity in the rat neostriatum: differential accumulation in projection and interneurons. Exp Neurol 144:239-247.

Landwehrmeyer G, McNeil S, Dure IV LS, Ge P, Aizawa H, Huang Q, Ambrose C, Duyao M, Bird E, Bonilla E, de Young M, Avila-Gonzales A, Wexler N, DiFiglia M, Gusella J, MacDonald M, Penney J, Young A, Vonsattel J-P (1995) Huntington's disease gene: regional and cellular expression in brain of normal and affected individuals. Ann Neurol 37:218-230.

Leterrier J, Rusakov D, Nelson B, Linden M (1994) Interactions be- tween brain mitochondria and cytoskeleton: evidence for specialized outer membrane domains involved in the association of cytoskeletonassociated proteins to mitochondria in situ and in vitro. Microsc Res Tech 27:233-261.

Li S-H, Li X-J (1998) Aggregation of the N-terminus of huntingtin depends on its length of glutamine repeat. Hum Mol Genet 7:777-782.

Li S-H, Schilling G, Young WS, Li X-J, Margolis R, Stine O, Wagster M, Abbott M, Franz M, Ranen N, Folstein S, Hedreen J, Ross C (1993) Huntington's disease gene (IT15) is widely expressed in human and rat tissues. Neuron 11:985-993.

Li S-H, Gutekunst CA, Hersch SM, Li X-J (1998a) Interaction of huntingtin-associated protein with dynactin P150 Glued. J Neurosci 18:1261-1269.

Li S-H, Gutekunst CA, Hersch SM, Li X-J (1998b) Association of HAP1 isoforms with a unique cytoplasmic structure. J Neurochem, in press.

Li X-J, Li S-H, Sharp A, Nucifora F, Schilling G, Lanahan A, Ross C (1995) A huntingtin-associated protein enriched in brain with implications for pathology. Nature 378:398-402.

Li X-J, Sharp A, Li S-H, Dawson T, Snyder S, Ross C (1996) Huntingtinassociated protein (HAP1): discrete neuronal localizations in the brain resemble those of neuronal nitric oxide synthase. Proc Natl Acad Sci USA 93:4839-4844.

Navarro A, Tolivia J, Alvarez-Uria M (1995) Ultrastructural study of a special type of ependymal cell at paraventricular level of the golden hamster third ventricle. Histol Histopathol 10:861-868.

Reynolds E (1963) The use of lead citrate at high $\mathrm{pH}$ as an electron opaque stain in electron microscopy. J Cell Biol 117:208-212.

Scherzinger E, Lurz R, Turmaine M, Mangiarini L, Hollenbach B, Hasenbank R, Bates GP, Davies SW, Lehrach H, Wanker EE (1997) Huntingtin-encoded polyglutamine expansions form amyloid-like protein aggregates in vitro and in vivo. Cell 90:549-558.

Sharp A, Loev S, Schilling G, Li S-H, Li X-J, Bao J, Wagster M, Kotzuk J, Steiner J, Lo A, Hedreen J, Sisodia S, Snyder S, Dawson T, Ryugo D, Ross C (1995) Widespread expression of Huntington's disease gene (IT15) protein product. Neuron 14:1065-1074.

Shinoda K, Mori S, Ohtsuki T, Osawa Y (1992) An aromataseassociated cytoplasmic inclusion, the "stigmoid body," in the rat brain: I. Distribution in the forebrain. J Comp Neurol 322:360-376.

Shinoda K, Nagano M, Osawa Y (1993) An aromatase-associated cytoplasmic inclusion, the "stigmoid body," in the rat brain: II. Ultrastructure (with a review of its history and nomenclature). J Comp Neurol 329:1-19.

Strong T, Tagle D, Valdes J, Elmer L, Boehm K, Swaroop M, Kaatz K, Collins F, Albin R (1993) Widespread expression of the human and rat Huntington's disease gene in brain and nonneural tissues. Nat Genet 5:259-265.

Takeuchi IK, Takeuchi YK (1982) Ultrastructural and cytochemical studies on nucleolus-like bodies in early postimplantation rat embryos. Cell Tissue Res 226:257-266.

Trembleau A, Morales M, Bloom F (1994) Aggregation of vasopressin mRNA in a subset of axonal swellings of the median eminence and posterior pituitary: light and electron microscopic evidence. J Neurosci 14:39-53.

Trottier Y, Devys D, Imbert G, Saudou F, An I, Lutz Y, Weber C, Agid Y, Hirsch E, Mandel J-L (1995) Cellular localization of the Huntington's disease protein and discrimination of the normal and mutated form. Nat Genet 10:104-110.

Vallee R (1982) A taxol-dependent procedure for the isolation of microtubules and microtubule-associated proteins (MAPs). J Cell Biol 92:435-442.

Wanker E, Rovira C, Scherzinger E, Hasenbank R, Walter S, Tait D, Colicelli J, Lehrach H (1997) HIP-1: a huntingtin interacting protein isolated by the yeast two-hybrid system. Hum Mol Genet 6:487-495.

Weakley B (1969) Basic protein and ribonucleic acid in the cytoplasm of the ovarian oocyte in the golden hamster. Z Zellforsch Mikrosk Anat 112:69-84.

Wellington CL, Brinkman RR, O'Kusky JR, Hayden MR (1997) Toward understanding the molecular pathology of Huntington's disease. Brain Pathol 7:979-1002.

Yi H, Hersch S (1997) Subcellular localization of receptors using electron microscopy. In: Receptor localization: laboratory methods and procedures (Ariano M, ed), pp 107-127. New York: Wiley. 